\section{ORIGINAL RESEARCH}

\section{Loumiotis}

P.I. D'Urso

R. Tawk

H.J. Cloft

D.F. Kallmes

V. Kairouz

R. Hanel

G. Lanzino

\title{
Endovascular Treatment of Ruptured Paraclinoid Aneurysms: Results, Complications, and Follow-Up
}

BACKGROUND AND PURPOSE: Paraclinoid aneurysms are an uncommon cause of aneurysmal SAH, and their treatment is challenging. To assess the effectiveness and safety of endovascular treatment of ruptured paraclinoid aneurysms, we performed a retrospective analysis of 33 patients.

MATERIALS AND METHODS: Clinical and radiologic information on 33 patients undergoing endovascular therapy between 1999 and 2010 was retrospectively reviewed. Angiographic results were evaluated with the modified Raymond grading system, whereas clinical outcomes were evaluated with the mRS scale.

RESULTS: Seventeen (52\%) aneurysms were classified as clinoid segment aneurysms, and 16 (48\%), as ophthalmic segment aneurysms. Twenty-six (79\%) aneurysms were small, 6 (18\%) were large, 1 was $(3 \%)$ giant, and $39 \%$ were wide-neck. Coiling was done with balloon assistance in $36 \%$ of cases and stent-assistance in $6 \%$. Technical complications occurred in 1 patient, contributing to death. Early clinical complications causing permanent disability occurred in 3\% of cases. One patient (3\%) had fatal rebleeding 18 days after treatment. Overall, procedure-related morbidity and mortality were, respectively, $3 \%$ and $6 \%$. Complete occlusion of the aneurysm was achieved in $36 \%$ of patients after initial treatment and in 65\% during follow-up (average, 29.3 months). Seven patients had recurrences requiring retreatment (30\%). Clinical outcome (average, 32.9 months) was good in $75 \%$ of patients and poor in $25 \%$. No delayed complications related to treatment and/or the aneurysm occurred.

CONCLUSIONS: Ruptured paraclinoid aneurysms are challenging lesions from an endovascular and surgical point of view. Despite the high rate of recurrences, good clinical results and protection against rebleeding can be achieved with current endovascular techniques.

ABBREVIATIONS: GCS = Glasgow Coma Scale; ISAT = International Subarachnoid Aneurysm Trial; $\mathrm{mRS}=$ modified Rankin Scale; WFNS $=$ World Federation of Neurosurgical Societies

$T^{1}$ he paraclinoid ICA location is common in patients with unruptured intracranial aneurysms. However, paraclinoid aneurysms are an uncommon cause of aneurysmal SAH, and in large series, these represent approximately $1.4 \%-9.1 \%$ of all patients with ruptured aneurysms. ${ }^{1-3}$ Because of their location in proximity to the skull base, surgery for paraclinoid aneurysms can be challenging and often requires extensive drilling of the anterior clinoid process and skull base to obtain proximal control and expose the aneurysm neck in its entirety. Because of these challenges, paraclinoid aneurysms have been one of the most common indications for endovascular treatment. ${ }^{4,5}$ There is extensive literature on endovascular treatment of unruptured paraclinoid aneurysms. However, due to their rarity, to our knowledge, little is known about the results and outcome of patients with ruptured paraclinoid aneurysms undergoing endovascular treatment. In this article, we summarize our experience with patients with ruptured paraclinoid aneurysms treated with endovascular techniques at our institutions.

\footnotetext{
Received April 20, 2011; accepted after revision June 21
}

From the Departments of Neurosurgery (I.L., P.I.D., G.L.) and Radiology (H.J.C., D.F.K.), Mayo Clinic, Rochester Minnesota; and Department of Neurosurgery (R.T., V.K., R.H.), Mayo Clinic, Jacksonville, Florida.

Please address correspondence to Giuseppe Lanzino, MD, Department of Neurosurgery, Mayo Clinic, 200 First St SW, Rochester, MN 55905; e-mail: Lanzino.Giuseppe@mayo.edu http://dx.doi.org/10.3174/ajnr.A2825

\section{Materials and Methods}

After approval of the institutional review board, a retrospective chart review of patients treated with coil embolization at our institutions (Mayo Clinic Rochester from 1999 to 2010 and Mayo Clinic Florida from 2007 to 2010) was undertaken. Thirty-four patients had ruptured aneurysms located in the paraclinoid region. This represents $9 \%$ of all ruptured aneurysms treated with endovascular techniques at Mayo Clinic Rochester between 1999 and 2010. Clinical and radiologic information were retrospectively abstracted from the chart. Patients with dissecting, fusiform, and blisterlike aneurysms were excluded. Similarly patients who presented with SAH from another aneurysm and had an unruptured paraclinoid aneurysm were excluded. Information collected included patient demographics, risk factors (including a history of ischemic cerebrovascular disease, smoking, hypertension, and a family history of intracranial aneurysms), and length of hospital stay. Clinical outcomes were reported by using the mRS scores, which were documented at baseline (before the SAH based on information collected on admission) and at the last available clinical follow-up. ${ }^{6,7}$ The mRS score has been considered a well-accepted measure of outcomes for aneurysm repair. ${ }^{8}$ A "good" outcome was defined as an mRS score of $0-2$; a "poor" outcome was defined as an mRS score of 3-6. The patient admission status was codified according to the WFNS score, and the GCS score was recorded. The amount of blood on CT was defined according to the Fisher grade. The total number of aneurysms identified on cerebral angiograms along with the location and size of each was analyzed. Paraclinoid aneurysms were classified according to Bouthillier et al. ${ }^{9}$ Transitional, carotid cave, posterior carotid wall, and superior hypo- 
physeal aneurysms were considered "clinoid segment" (C5) aneurysms; and ophthalmic aneurysms, "ophthalmic segment" (C6) aneurysms.

Information about treatment, including the need for balloon assistance or stent assistance, was collected. Stents became available at both centers in September 2002. Technical complications including perforation, coil prolapse, coil migration, endoluminal thrombus formation, and distal emboli were recorded. Coil prolapse was subclassified into no flow-limiting and flow-limiting, and information was acquired about whether systemic and/or intra-arterial administration of glycoprotein IIB/IIIA inhibitors (eptifibatide [Integrilin] or abciximab [ReoPro]) was used. Early neurologic complications were defined as any thromboembolic or hemorrhagic complication occurring within 1 month. SAH-related complications such as vasospasm or hydrocephalus were also recorded. Non-neurologic complications, including access site hematoma (defined as any hematoma requiring prolonged immobilization, blood transfusion, or surgical repair), were recorded. Aneurysm occlusion was graded by using a 3-point mRS. ${ }^{10}$

Radiologic follow-up information for every aneurysm was recorded at various intervals and at the last radiologic follow-up. We prescribe routine follow-up imaging at 6 months in all patients with ruptured aneurysms, but earlier angiography is sometimes performed if there is concern for early recurrence. Specifically, we recorded occlusion rates and whether conventional angiography or MRA studies were performed. Information was also extracted about whether the aneurysm had been retreated and the occlusion rate after retreatment. Clinical information collected at the last follow-up included that regarding any occurrence of rebleeding, delayed ischemic symptoms related to possible thromboembolism induced by the coils and/or stent, mRS score, and, if applicable, the cause of worsening. Rates of balloon-assisted and stent-assisted coiling, immediate angiographic obliteration, and recurrence were compared with those encountered in the overall institutional experience with endovascular treatment of ruptured aneurysms in other locations. Data about postprocedural single or double antiplatelet therapy (aspirin and/or clopidogrel) were considered. The institutional policies regarding antiplatelet medication for ruptured aneurysms are generally not to routinely use periprocedural aspirin in ruptured aneurysms. However, aspirin and clopidogrel are administered when using adjunctive stents. For these comparisons, only Mayo Clinic Rochester data were used because overall data from Mayo Clinic Florida were not available.

The $\chi^{2}$ test was used for comparison of proportions, and the 2 -sided $t$ test, for comparison of means. Results were considered significant for $P$ values $\leq .05$.

Patients treated in the first half of the study period (from 1999 to 2004) were compared with patients treated during the second half (from 2005 to 2010). Continuous data are presented as mean \pm SD. All statistical analyses were performed with JMP software, Version 9.0.1 (SAS Institute, Cary, North Carolina).

\section{Results}

Thirty-three patients were admitted with SAH from aneurysms located in the paraclinoid region. Twenty-six patients (79\%) were treated within 24 hours from the SAH; 2 patients (6\%), after 24 and within 48 hours; and 5 (15\%), after 48 hours. Demographic and clinical data of these patients are summarized in Table 1. Twenty-six (79\%) aneurysms were small ( $<10 \mathrm{~mm}), 6(18 \%)$ were large $(10$ to $25 \mathrm{~mm})$, and 1 (3\%) was giant $(>25 \mathrm{~mm})$ (Table 2$)$. The mean size of the

\begin{tabular}{lc}
\hline Table 1: Patients characteristics & \\
\hline & $\begin{array}{c}\text { Ruptured } \\
\text { Paraclinoid }\end{array}$ \\
\hline Sex & \\
F & $27(82 \%)$ \\
M & $6(18 \%)$ \\
F/M & 4.5 \\
Mean age (yr) & $52.9 \pm 14.3$ \\
Risk factors & \\
Hypertension & $41 \%$ \\
Diabetes mellitus & $3 \%$ \\
Tobacco abuse & \\
$\quad$ Current & $55 \%$ \\
Prior & $3 \%$ \\
Personal history of stroke & $9 \%$ \\
Family history of intracranial aneurysm & $11 \%$ \\
History of SAH & $3 \%$ \\
Pretreatment mRS (before SAH) & \\
0 & $52 \%$ \\
1 & $42 \%$ \\
3 & $3 \%$ \\
4 & $3 \%$ \\
Interval between SAH and treatment & \\
$<24 \mathrm{Hr}$ & \\
$\geq 24 \leq 48 \mathrm{Hr}$ & $26(79 \%)$ \\
$\geq 48 \mathrm{Hr}$ & $2(6 \%)$ \\
Length of hospital stay (days) & $5(15 \%)$ \\
\hline & $16.1 \pm 11.9$ \\
\hline
\end{tabular}

aneurysm fundus was $8.17 \pm 6.11$, and the mean size of the aneurysm neck was $3.88 \pm 1.89$. Thirteen (39\%) aneurysms were wide-neck (neck $>4 \mathrm{~mm}$ ). Forty-seven percent of patients had multiple aneurysms, and $9(27 \%)$ patients had bilateral paraclinoid aneurysms. Only in 1 case could a history of $\mathrm{SAH}$ from rupture of another aneurysm be elicited. Table 3 summarizes clinical and CT findings on admission.

Fifteen (45\%) patients were treated between 1999 and 2004, and 18 (55\%), between 2005 and 2010. No significant differences were observed within these subgroups in relation to aneurysm size $(P=.669)$ and aneurysm neck size $(P=.545)$.

\section{Treatment}

All aneurysms were treated with reconstructive therapy. Twenty-six aneurysms required only 1 endovascular procedure. Because of recurrence, 6 aneurysms required 2 procedures, and 1 aneurysm, 3. Recurrences were managed with recoiling in 5 patients and flow-diverter deployment in 2 patients. No significant difference between recurrences and postprocedural use of antiplatelet medication was observed $(P=.942)$. The recurrence rate in this population was $30 \%$, higher than that of ruptured aneurysms in other locations observed in our endovascular series (30\% versus $18 \%, P=.166$, Mayo Clinic Rochester dataset). Twelve patients (36\%) underwent balloon-assisted coiling, 7 of whom had broad-neck aneurysms, while in 2 patients $(6 \%)$, the procedure was stentassisted; these percentages are significantly higher compared with those of balloon- and stent-assistance observed in our endovascular series of ruptured aneurysms in other locations (34\% versus $15 \%, P=.015$, Mayo Clinic Rochester dataset). 


\begin{tabular}{lc}
\hline Table 2: Distribution of the subtypes of paraclinoid aneurysm and size \\
\cline { 2 - 2 } Aneurysm Location & Small $(<10 \mathrm{~mm})$ \\
\hline Clinoid segment (C5) & 15 \\
Ophthalmic segment (C6) & 11 \\
\hline \multicolumn{2}{l}{} \\
\hline Table 3: Clinical and radiologic admission grade \\
\hline Scale & Grade \\
\hline WFNS & \\
I & $20(61 \%)$ \\
II & $4(12 \%)$ \\
III & $2(6 \%)$ \\
IV & $3(9 \%)$ \\
V & $4(12 \%)$ \\
Fisher Grade & \\
1 & $3(9 \%)$ \\
2 & $4(12 \%)$ \\
3 & $17(52 \%)$ \\
4 & $9(27 \%)$ \\
\hline
\end{tabular}

\section{Periprocedural Complications}

In 1 patient (3\%), a 47-year-old with a grade V SAH from a very large carotid-ophthalmic aneurysm, aneurysm perforation occurred during coiling. The patient died as a result of this complication combined with the effects of the primary SAH.

\section{Clinical Complications}

Early clinical complications (related either to the procedure or to $\mathrm{SAH}$ ), causing transient neurologic symptoms and signs, occurred in 6 patients $(18 \%)$, while permanent disability occurred only in 1 patient (3\%). Transient neurologic worsening was related to hydrocephalus (3 patients), vasospasm (3 patients, with 1 patient having neurologic worsening attributed to both vasospasm and hydrocephalus). Permanent disability was observed in 1 patient admitted with a Fisher grade $4 \mathrm{SAH}$ and associated intraparenchymal and subdural hematoma, both requiring surgical evacuation after coiling.

Mortality following treatment within 30 days after admission and not related to technical complications occurred in 2 patients $(6 \%)$. One patient had a rebleeding 18 days after complete coiling of a posterior carotid wall aneurysm; another patient, admitted with a poor-grade SAH, died the day following admission from complications related to the $\mathrm{SAH}$.

In conclusion, procedural mortality was $3 \%$, and the rebleeding rate following endovascular treatment was 3\%, while there was no permanent morbidity attributed to treatment. However, as previously specified, 1 patient (3\%) had permanent morbidity from sequelae of the original bleed.

\section{Angiographic Outcome}

All patients had immediate postoperative angiography, which demonstrated complete aneurysm obliteration (class 1 ) in 12 patients $(36 \%)$; this rate was lower compared with that observed in ruptured aneurysms in other locations (31\% versus $43 \%, P=.194$, Mayo Clinic Rochester dataset).

Radiologic follow-up information was available for 14 patients within 6 months and for 12 patients within 12 months, while in 13 patients, radiologic follow-up longer than 1 year was available, with some patients having multiple angiographic follow-ups at various intervals (Table 4). In 66\% of

\begin{tabular}{|c|c|c|c|c|}
\hline $\begin{array}{l}\text { Occlusion } \\
\text { Grade }\end{array}$ & Immediate & $6 \mathrm{Mo}$ & $12 \mathrm{Mo}$ & $\begin{array}{c}\text { Last } \\
\text { Follow-Up }\end{array}$ \\
\hline Class 1 & $12 / 33(36 \%)$ & $7 / 14(50 \%)$ & $5 / 12(42 \%)$ & $9 / 13(69 \%)$ \\
\hline Class 2 & $16 / 33(48 \%)$ & $5 / 14(36 \%)$ & $6 / 12(50 \%)$ & $4 / 13(31 \%)$ \\
\hline Class 3 & $5 / 33(15 \%)$ & $2 / 14(14 \%)$ & $1 / 12(8 \%)$ & - \\
\hline
\end{tabular}

cases, angiographic follow-up was obtained with DSA, while in $34 \%$ of cases, with MRA. The mean length of radiologic follow-up was $29.3 \pm 26.7$ (range, 2-94 months).

After subgroup analysis, 6/15 (40\%) patients treated between 1999 and 2004 and 6/18 (33\%) patients treated between 2005 and 2010 had complete aneurysm occlusion immediately following endovascular treatment $(P=.692)$. Six of $11(55 \%)$ patients treated between 1999 and 2004 and 9/12 (75\%) treated between 2005 and 2010 had complete occlusion at follow-up $(P=.301)$. The higher rate of complete occlusion in the second half of the study was related to the higher chance of complete occlusion achieved in patients treated with balloonassistance between 2005 and $2010(P=.022)$.

Seven patients required retreatment for recurrences deemed to be clinically important in relation to the increased risk of rebleeding (Fig 1). Overall, at the end of the radiologic follow-up period, complete obliteration (including retreatment) was achieved in 16 of 24 patients (67\%) (Tables 5 and 6). No significant differences in recurrence rate were observed between the 1999-2004 and the 2005-2010 subgroups (20\% versus $22 \%, P=.876$ ).

\section{Clinical Outcome and Long-Term Morbidity}

Clinical follow-up longer than 3 months was available in 26 of the 30 survivors (average length of clinical follow up, $32.9 \pm$ 26.4 months; range, 3-94 months). Three patients died. A 47year-old man, admitted with a grade V SAH from a very large carotid-ophthalmic aneurysm, experienced aneurysm perforation during coiling and died due to the combined effects of the primary bleed and the periprocedural rupture. A 48-yearold man had rebleeding 18 days after the procedure despite initial complete angiographic occlusion of a posterior carotid wall aneurysm. The third patient, a 64-year-old woman admitted with a poor-grade SAH from a small posterior carotid wall aneurysm, died the day following the treatment from complications related to the SAH.

Of the patients available for follow-up, 22/26 (85\%) had a good outcome, while $4 / 26$ patients $(15 \%)$ had an overall poor outcome. In 2 patients, permanent disability was related to $\mathrm{SAH}$, while 2 patients recovered to their pre-SAH baseline but they had pre-existing (due to the SAH) disabilities which affected the follow-up mRS score. Time elapsing between SAH and aneurysm treatment was not significantly related to overall outcome $(P=.250)$. No cases of delayed worsening or death occurred during follow-up. No late $(>1$ month after treatment) rebleedings were reported. When patients who 

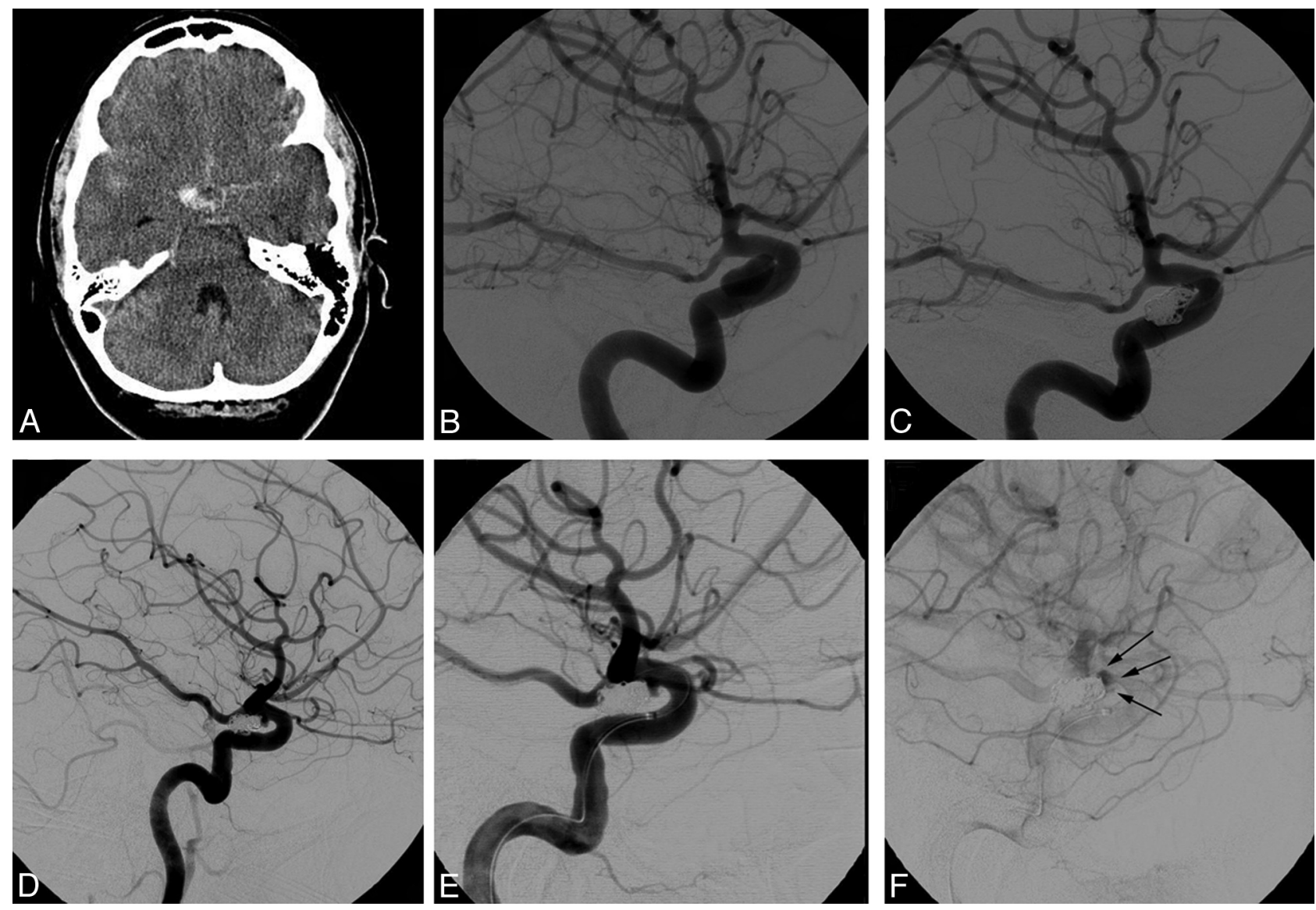

Fig 1. $A$, This 50-year-old woman was admitted with a WFNS grade I SAH with a thick clot localized around the right carotid cistern (axial noncontrast CT scan). $B$ and $C$, She was found to have a 9-mm elongated right superior hypophyseal aneurysm ( $B$, oblique projection) for which coil embolization was performed with near-complete occlusion ( $C$, oblique projection). $D$, A follow-up DSA 6 months later showed recurrence of the aneurysm (oblique projection). The recurrence was treated with a Pipeline Embolization Device (PED, Chestnut Medical Technologies, Menlo Park, California). $E$ and $F$, Oblique projections, early arterial phase $(E)$ and late venous phase $(A)$ after PED deployment, show stasis of contrast within the recurrent portion of the neck.

\begin{tabular}{lrc}
\hline \multicolumn{2}{l}{ Table 5: Angiographic outcome $^{\text {a }}$} \\
\hline $\begin{array}{lr}\text { Occlusion Grade } \\
\text { (Raymond class) }\end{array}$ & Immediate & Follow-Up \\
\hline Class 1 & $7 / 23(30 \%)$ & $15 / 23(65 \%)$ \\
Class 2 & $13 / 23(57 \%)$ & $7 / 23(31 \%)$ \\
Class 3 & $3 / 23(13 \%)$ & $1 / 23(4 \%)$ \\
\hline
\end{tabular}

Occlusion grade scored with Raymond class

a Comparative results between immediate and delayed angiographic occlusion grade in a restricted subgroup of patients with radiology at any time during the follow-up.

\begin{tabular}{lcc}
\hline Table 6: Number of retreatments $^{\mathbf{a}}$ & \\
\hline Within $6 \mathrm{Mo}$ & Within $12 \mathrm{Mo}$ & At the Last Follow-Up \\
3 & 2 & 2 \\
\hline
\end{tabular}

a Retreatment interval since initial intervention for 7 patients during the follow-up.

died within the first month of treatment are included, overall rates of good and poor outcome were 75\% (22/29) and 25\% (7/29), respectively.

\section{Discussion}

We report our experience with ruptured paraclinoid aneurysms treated with endovascular embolization. In our series, complete aneurysm occlusion was achieved in 35\% of cases at the end of the original procedure. Eventually, complete angiographic obliteration (including those patients requiring retreatment) was achieved in $67 \%$ of patients at the last angio- graphic follow-up (range, 3-94 months). Subgroup analysis of patients treated in the first-and second-half intervals of our study showed that higher rates of aneurysm occlusion were observed in recent years, and this difference was related to a higher incidence of complete occlusion achieved in the second half of the study period in those patients treated with balloon assistance (despite the incidence of balloon-assisted coiling being similar in the 2 periods). This difference suggests a positive role of experience with this adjunctive technique in achieving better packing attenuation as well as a possible positive role of better coil designs in the latter part of the series. Although better packing attenuation and complete occlusion rates may be achieved with stent-assisted coiling, only 2 patients in the present series were treated with such techniques. In general, we try to avoid stent-assisted coiling in patients with ruptured aneurysms because of the need for dual antiplatelet therapy and the higher incidence of complications. ${ }^{11}$

Approximately $40 \%$ of the aneurysms treated had a wide neck ( $\geq 4 \mathrm{~mm}$ ), which may explain the low rate of immediate angiographic occlusion compared with other series. Wideneck aneurysms are a challenge for an endovascular approach and may require balloon or stent assistance to accomplish satisfactory anatomic results in terms of occlusion. We use balloon- or stent-assisted coiling selectively. In our experience, balloon and stent assistance $(36 \%$ and $6 \%$, respectively, total 
$42 \%$ ) were more commonly used in the treatment of ruptured paraclinoid aneurysms than in the treatment of ruptured aneurysms in other locations. Sherif et $\mathrm{al}^{12}$ analyzed a series of ruptured paraclinoid aneurysms treated with embolization and achieved complete obliteration in $76.3 \%$ of the aneurysms on the immediate postoperative angiograms and $82.9 \%$ at follow-up. However, only $21 \%$ of their patients had wide-neck aneurysms compared with $40 \%$ in our series, and this difference may partially explain the discrepancy in the immediate obliteration rates between the 2 series.

Repeat embolization because of insufficient obliteration was performed in 7 patients (30\%), most whom were retreated within 12 months of the first embolization procedure. This rate is higher compared with the recurrence rate observed in ruptured aneurysms in other locations of our series. The availability of flow diverters will most likely improve the rate of complete angiographic obliteration. We foresee a near-future in which ruptured paraclinoid aneurysms are treated with current endovascular techniques in the acute phase to achieve acceptable protection against immediate rebleeding. A few weeks later, once patients are past the acute phase, flow diverters will be used to supplement the original treatment and increase the likelihood of complete angiographic occlusion.

One of the main reasons for treating acutely ruptured aneurysms causing SAH is to "secure" these aneurysms and protect the patient from subsequent bleeding that could be fatal. In our series, early rebleeding following successful coil embolization occurred in 1 patient with a posterior carotid wall aneurysm. Although the aneurysm was judged as completely coiled at the end of the procedure, rebleeding occurred 18 days after the procedure. The rebleeding rate observed in our series is comparable with that reported from the ISAT study (early rebleeding rate, $1.9 \%) .{ }^{13}$ More recent studies have suggested negligible rates of early and midterm rebleeding, ${ }^{1,14}$ implying that with increasing experience and improved devices, the rate of early rebleeding after coil embolization may be decreasing. In accordance with the ISAT study, coil embolization confers long-term protection because no rebleeding was observed after a mean clinical follow-up of 28 months. ${ }^{13}$

Rates of neurologic morbidity and mortality related to the procedure have progressively improved after coil embolization of ruptured aneurysms. Our series confirms the safety of the endovascular strategy, even in the case of challenging and often wide-neck paraclinoid aneurysms, which often require advanced endovascular techniques. One patient had an intraprocedural rupture following perforation of a giant paraclinoid aneurysm and died as a result of the effects of the original hemorrhage (his WFNS score was V on admission) combined with the deleterious effects of the perforation. No other permanent morbidity was observed. In analyzing the results of this series, one should consider that patients treated with endovascular techniques were not ideal surgical candidates because of aneurysm- and patient-related factors and, therefore, represent a selected high-risk population not necessarily representative of the overall population of patients with ruptured paraclinoid aneurysms seen at our institution. Our results are in line with those in other studies on the outcome after endovascular treatment of paraclinoid aneurysms, reporting procedure-related morbidity and mortality rates ranging between $3 \%$ and $8.3 \%$, and $0 \%$ and $1.5 \%$, respectively (though most of these studies included mainly patients with unruptured aneurysms). ${ }^{4,12,15-18}$ Our series confirms the observation that multiple (and often bilateral) aneurysms are present in patients with paraclinoid aneurysms. This association is probably related to the fact that development of paraclinoid aneurysms can be related to an intrinsic weakness of the carotid wall in this specific region, which, in turn, predisposes patients to mirror aneurysms.

Our study has some limitations related to its retrospective nature and the variable follow-up (common to most series dealing with ruptured aneurysms because many of these patients have limited resources and are often noncompliant with follow-up recommendations). In addition, the population studied is a selected group of patients deemed not to be ideal surgical candidates, and we do not have the overall denominator of patients from which the pool described is obtained. Nevertheless, unlike most other series on paraclinoid aneurysms in the literature, this study gives a snapshot of current endovascular results for ruptured paraclinoid aneurysms and provides a "modern" comparison against which more advanced endovascular methods can be compared.

\section{Conclusions}

Although unruptured aneurysms of the paraclinoid carotid artery are very common, these aneurysms represent only a minority of aneurysms presenting with SAH. Ruptured paraclinoid aneurysms are challenging lesions from an endovascular and surgical point of view. Acceptable clinical outcomes and protection against rebleeding can be achieved with current endovascular techniques, though retreatment is not uncommon due to clinically significant recurrences. Given the protection against rebleeding in the acute phase with coil embolization alone, it is conceivable that in the near future, more and more patients will be treated with a "staged" endovascular strategy of coil embolization in the acute phase after SAH followed by a second stage during which a flow diverter is placed across the coiled aneurysm to ensure higher rates of complete obliteration.

Disclosures: Harry J. Cloft—RELATED: Grant. MicroVention, Comments: enrolling center for Gel the Neck registry sponsored by MicroVention; UNRELATED: Board Membership: Medtronic, Comments: serve on the Data Safety and Monitoring Board for the Kyphoplasty and Vertebroplasty in the Augmentation and Restoration of Vertebral Body Compression Fractures trial; Grants/Grants Pending. Cordis, ${ }^{*}$ Mindframe. * David Kallmes-UNRELATED: Royalties: University of Virginia patent foundation, Comments: spine-fusion device; Payment for Development of Educational Presentations: eV3, ${ }^{*}$ CareFusion. ${ }^{*}$ Ricardo HanelUNRELATED: Board Membership: Neurovasx, Comments: scientific advisory board; Payment for Lectures, Including Service on Speaker Bureaus: Codman; Other. eV3, ${ }^{*}$ Comments: support travel expenses for Pipeline training. Giuseppe Lanzino-UNRELATED: Expert Testimony. Chestnut Medical/Covidien, ${ }^{*}$ Comments: testified in front of FDA panel for approval of Pipeline embolization device; Grants/Grants Pending. eV3, ${ }^{*}$ Synthes, ${ }^{*}$ Comments: testified in front of FDA panel for approval of Pipeline embolization device. *Money to the institution.

\section{References}

1. Molyneux A, Fox A, Sneade M, et al. Cerecyte coil trial: the angiographic outcome of endovascular coiling in patients with ruptured and unruptured intracranial aneurysms treated with Cerecyte compared with bare platinum coils-Results of a prospective randomized trial. J Neurointervent Surg 2010; 2:A7

2. Nguyen TN, Raymond J, Guilbert F, et al. Association of endovascular therapy of very small ruptured aneurysms with higher rates of procedure-related rupture. J Neurosurg 2008;108:1088-92

3. Park HK, Horowitz M, Jungreis C, et al. Periprocedural morbidity and mor- 
tality associated with endovascular treatment of intracranial aneurysms. AJNR Am J Neuroradiol 2005;26:506-14

4. Park HK, Horowitz M, Jungreis C, et al. Endovascular treatment of paraclinoid aneurysms: experience with 73 patients. Neurosurgery 2003;53:14-23, discussion 24

5. Lanzino G, Guterman L, Hopkins L. Endovascular treatment of aneurysms. In: Winn HR, ed. Youmans Neurological Surgery. 5th ed. Philadelphia: Saunders; 2004:2057-78

6. Bonita R, Beaglehole R. Recovery of motor function after stroke. Stroke 1988;19:1497-500

7. Rankin J. Cerebral vascular accidents in patients over the age of 60. II. Prognosis. Scott Med J 1957;2:200-15

8. Meyers PM, Schumacher HC, Higashida RT, et al. Reporting standards for endovascular repair of saccular intracranial cerebral aneurysms. Stroke 2009; 40:e366-79

9. Bouthillier A, van Loveren HR, Keller JT. Segments of the internal carotid artery: a new classification. Neurosurgery 1996;38:425-32, discussion 432-33

10. Roy D, Milot G, Raymond J. Endovascular treatment of unruptured aneurysms. Stroke 2001;32:1998-2004

11. Bodily KD, Cloft HJ, Lanzino G, et al. Stent-assisted coiling in acutely ruptured intracranial aneurysms: a qualitative, systematic review of the literature. AJNR Am J Neuroradiol 2011;32:1232-36. Epub 2011 May 5
12. Sherif C, Gruber A, Dorfer C, et al. Ruptured carotid artery aneurysms of the ophthalmic (C6) segment: clinical and angiographic long term follow-up of a multidisciplinary management strategy. J Neurol Neurosurg Psychiatry 2009; 80:1261-67

13. Molyneux A, Kerr R, Stratton I, et al. International Subarachnoid Aneurysm Trial (ISAT) of neurosurgical clipping versus endovascular coiling in 2143 patients with ruptured intracranial aneurysms: a randomised trial. Lancet 2002;360:1267-74

14. Spetzler R. Three-year follow-up results of BRAT. In: Proceedings of the Annual Meeting of the American Association of Neurological Surgeons, Denver, Colorado. April 9-13, 2011

15. Hoh BL, Carter BS, Budzik RF, et al. Results after surgical and endovascular treatment of paraclinoid aneurysms by a combined neurovascular team. $\mathrm{Neu}$ rosurgery 2001;48:78-89, discussion 89-90

16. Jin SC, Kwon do H, Ahn JS, et al. Clinical and radiological outcomes of endovascular detachable coil embolization in paraclinoid aneurysms: a 10-year experience. J Korean Neurosurg Soc 2009;45:5-10. Epub 2009 Jan 31

17. Roy D, Raymond J, Bouthillier A, et al. Endovascular treatment of ophthalmic segment aneurysms with Guglielmi detachable coils. AJNR Am J Neuroradiol 1997;18:1207-15

18. Thornton J, Aletich VA, Debrun GM, et al. Endovascular treatment of paraclinoid aneurysms. Surg Neurol 2000;54:288-99 